\title{
SOLAR INTERNAL ROTATION
}

\author{
JESPER SCHOU AND THE SOI INTERNAL ROTATION TEAM \\ HEPL Annex A201 \\ Stanford, CA 94305-4085 \\ $U S A$
}

\begin{abstract}
.
With the flood of high quality helioseismic data from the instruments on the SOHO spacecraft (MDI/VIRGO/GOLF) and ground based instruments (e.g. GONG, LOWL, BiSON, IRIS, MWO-CrAO and TON) we have been able to get increasingly detailed information on the rotation and other large scale flows in the solar interior.

Here we will discuss some of the highlights of what we have learned so far and what we may expect to learn in the near future. Among the recent advances have been tighter constraints on the tachocline at the bottom of the convection zone and detection of details in the surface rotation rate similar to the torsional oscillations found in the surface Doppler shift.
\end{abstract}

\section{Introduction}

The determination of the solar internal rotation has been one of the central problems in helioseismology and several significant results have been obtained (see e.g. Duvall et al., 1984; Christensen-Dalsgaard \& Schou, 1988; Tomczyk, Schou \& Thompson, 1995; Thompson et al., 1996 and references therein). While substantial theoretical efforts have been made to understand the origin of the solar differential rotation, no clear understanding of underlying mechanisms has yet emerged. It is widely believed that an understanding of the differential rotation is a key element in understanding the solar cycle. For these and other reasons it is important to continue to constrain the solar internal rotation further.

In this review we will briefly discuss some of the recent helioseismic results concerning solar rotation. We shall not go into any technical details but rather refer the interested reader to the latest papers describing the results, in particular Schou et al. (1998), Kosovichev \& Schou (1997) and Basu (1997). Details of the various inversion methods can be found in Thompson (1998). 
Global flows other than rotation (e.g. meridional flows) will not be discussed here. Giles et al. (1997) and Giles \& Duvall (1998) discuss some findings on meridional flows from time-distance helioseismology.

\section{Global Picture}

Most of the results presented here are from 144 days of MDI Medium- $l$ data from May 9, 1996 to September 29, 1996. The Medium- $l$ program is designed to cover the range $0 \leq l \leq 300$, where $l$ is the spherical harmonic degree. For this study only $\mathrm{p}$ modes with degree up to $\approx 200$ and $\mathrm{f}$ modes up to 250 have been used, corresponding to the modes that are well separated in the power spectra.

The leading-order effect of solar rotation is to split the $(2 l+1)$ modes in a single $(n, l)$ (with $n$ being the radial order of the modes) multiplet by an amount (Schou et al., 1994)

$$
\Delta \omega_{n l m} \equiv \omega_{n l m}-\omega_{n l}=\int_{0}^{R} \int_{0}^{\pi} K_{n l m}(r, \theta) \Omega(r, \theta) r d r d \theta
$$

where $m$ is the azimuthal order of the mode, $R$ is the solar radius, $K_{n l m}$ is a mode kernel (that can be calculated from a solar model), $\Omega$ is the rotation rate and $\omega_{n l}$ is the ( $m$-independent) unperturbed mode frequency.

Unfortunately it is difficult to measure the splittings for individual modes and the $m$-dependence of the splittings is generally expanded using so-called $a$-coefficients in order to stabilize the fit:

$$
\Delta \omega_{n l m} / 2 \pi \equiv \Delta \nu_{n l m}=\sum_{i=1}^{i_{\max }} a_{i}(n, l) P_{i}^{(l)}(m)
$$

where the $P_{i}^{(l)}$ are suitably chosen polynomials of degree $i$ and the $a_{i}$ are the $a$ coefficients (Schou et al., 1994). For the present dataset the multiplets were fitted with $i_{\text {max }}=6, i_{\max }=18$ and $i_{\max }=36$. For each multiplet one of the three fits was chosen based on $l$ and the convergence of the fits. In most cases $i_{\text {max }}=36$ was used.

Figure 1 shows the rotation rate in the solar interior inferred from the $144 \mathrm{~d}$ dataset using a Regularized Least Squares (RLS) inversion method (Schou et al., 1994). Other methods, such as optimally localized averages methods and so-called $1.5 \mathrm{~d}$ and $1 d \times 1 d$ methods, have also been applied to this dataset, but for lack of space, only the results from the RLS method are presented in this review. The RLS method is currently the one for which most experience exists, but most of the other methods show very similar rotation profiles in the regions for which the methods produce reliable results. For more results and a discussion of the different methods see Schou et al. (1998) and Thompson (1998).

Several features stand out in Fig. 1: the differential rotation in the convection zone, the transition to (near) solid body rotation at the bottom of the convection zone, the steep gradient close to the surface and the jet in the convection zone near the pole. Some of these features have been seen before (e.g. Christensen-Dalsgaard \& Schou, 1988; Tomczyk, Schou \& Thompson, 1995; Thompson et al., 1996), but some are new and others have been substantially better resolved.

The large scale differential rotation in the convection zone has, of course, been seen before in numerous studies, as mentioned above. Again it appears that the rotation rate is closer to being constant on cones of constant latitude rather than constant 


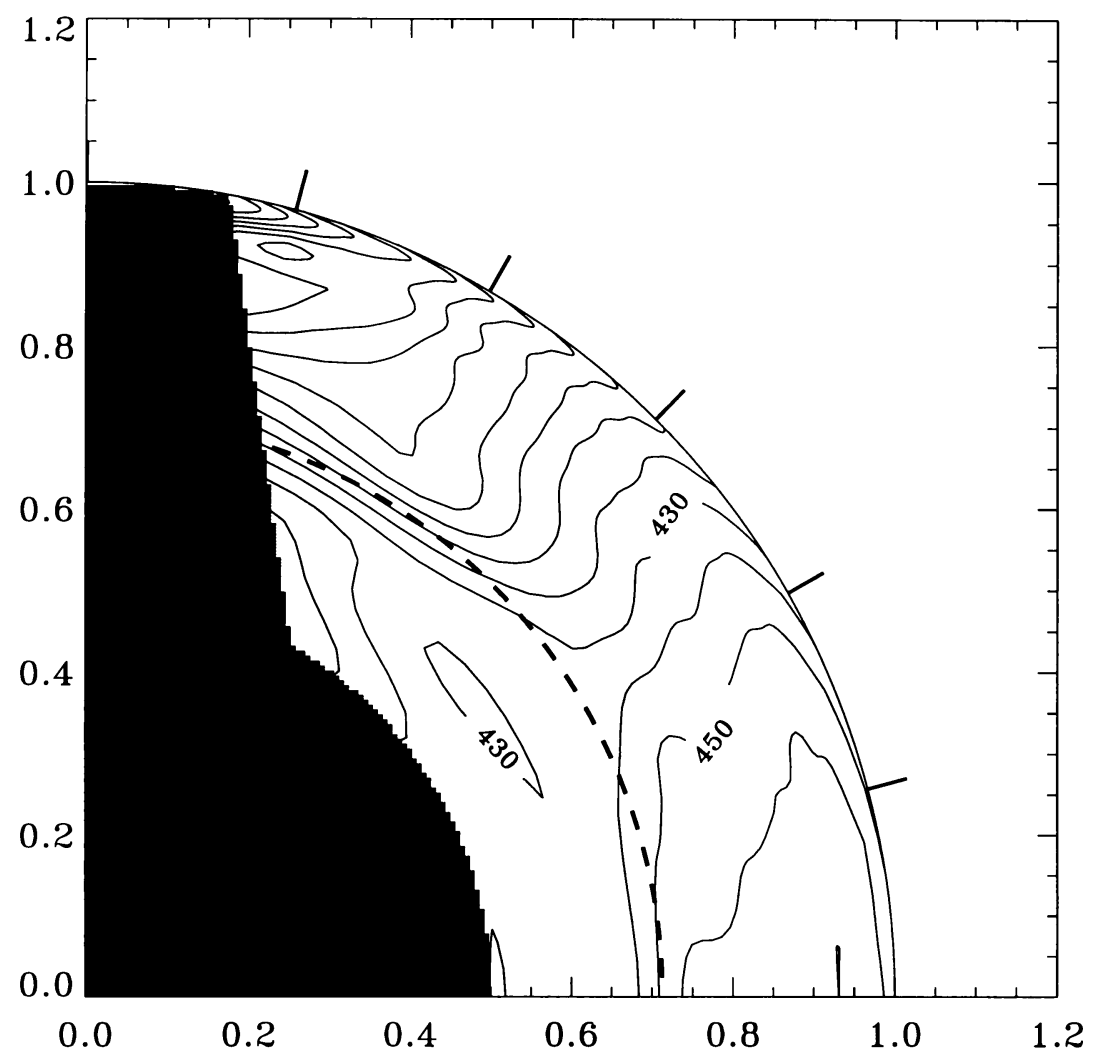

Figure 1. Contour plot of the solar rotation $(\Omega / 2 \pi(\mathrm{nHz}))$. The input data were $a$-coefficients (up to $a_{35}$ ) from 144 days of MDI Medium-l data. The rotation rate in the region close to the rotation axis and close to the center could not be reliably determined from this dataset. This inversion was done using a regularized least squares method. Adapted from Schou et al. (1998).

on cylinders, contrary to earlier theoretical predictions. There are however significant deviations from this general behaviour, as outlined below.

The steep transition (tachocline) between the radiative interior and the convection zone has been seen before (Basu, 1997; Kosovichev, 1996; Charbonneau et al., 1997). Unfortunately inversions, such as the one shown in Fig. 1, are not able to resolve this region. To constrain the properties (e.g. width and position) of this region it is thus necessary to make further assumptions. One popular assumption is that the transition is well represented by a function with a few adjustable parameters. Kosovichev (1996) and Charbonneau et al. (1997) used an error function with adjustable position, height and width. Basu (1997) used a different parametrization of the transition. In all the cases shown here a width of zero would correspond to an abrupt step, but otherwise the numerical values of the widths are not directly comparable. Typically the fit was 


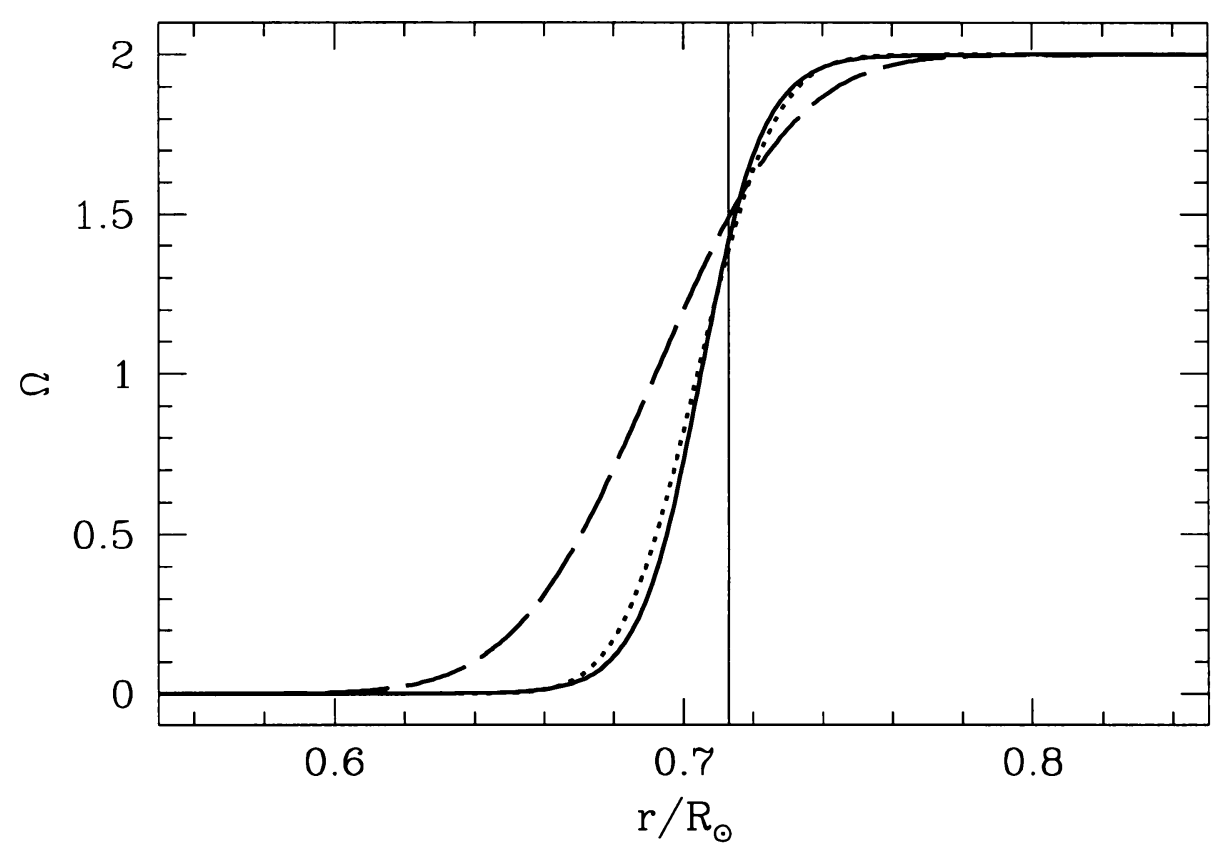

Figure 2. Rotation rate across the tachocline. Solid line from Basu (1997) using BBSO/GONG data. Dashed line from Kosovichev (1996) using BBSO data. Dotted line from Charbonneau et al. (1997) using LOWL data. The vertical solid line shows the bottom of the convection zone. The functions fitted were not the same in all cases. All studies used the $a_{3}$ coefficient in the fitting. The rotation profiles were rescaled such as to make the asymptotic value 0 in the deep interior and 2 in the convection zone.

carried out only for one measured quantity, in the cases shown here to the $a_{3}$ term in the expansion (1) which is related to the first non-constant term in the expansion of $\Omega$ with latitude.

A number of such fits are shown in Fig. 2 (adapted from Basu (1997)). The Charbonneau et al. and Basu fits are quite similar with both quoting widths different from zero by about $4 \sigma$. The Kosovichev result for the width is different from zero by just over $2 \sigma$, and is thus only marginally different from the others. It does, however, appear that the transition is not a step function and that most of the gradient is within the radiative interior.

A quite striking but possibly less reliable feature is the jet seen at 75 degrees latitude about $5 \%$ below the solar surface. This is more clearly seen in Figure 3 which also shows that it is quite statistically significant. This jet has a magnitude of around $20 \mathrm{nHz}$ (corresponding to about $20 \mathrm{~m} / \mathrm{s}$ ), a width of a few percent in radius and an extent of a few degrees in latitude. It should, however, be remarked that this feature is close to the edge of the reliable region and that other inversion methods show it less clearly. Also the resolution, especially in the latitude direction, is comparable to the extent of the feature, and therefore the jet may be narrower (and correspondingly faster) than the inversion seems to show. More $a$-coefficients or individual splittings would be needed to resolve this and other features better. 


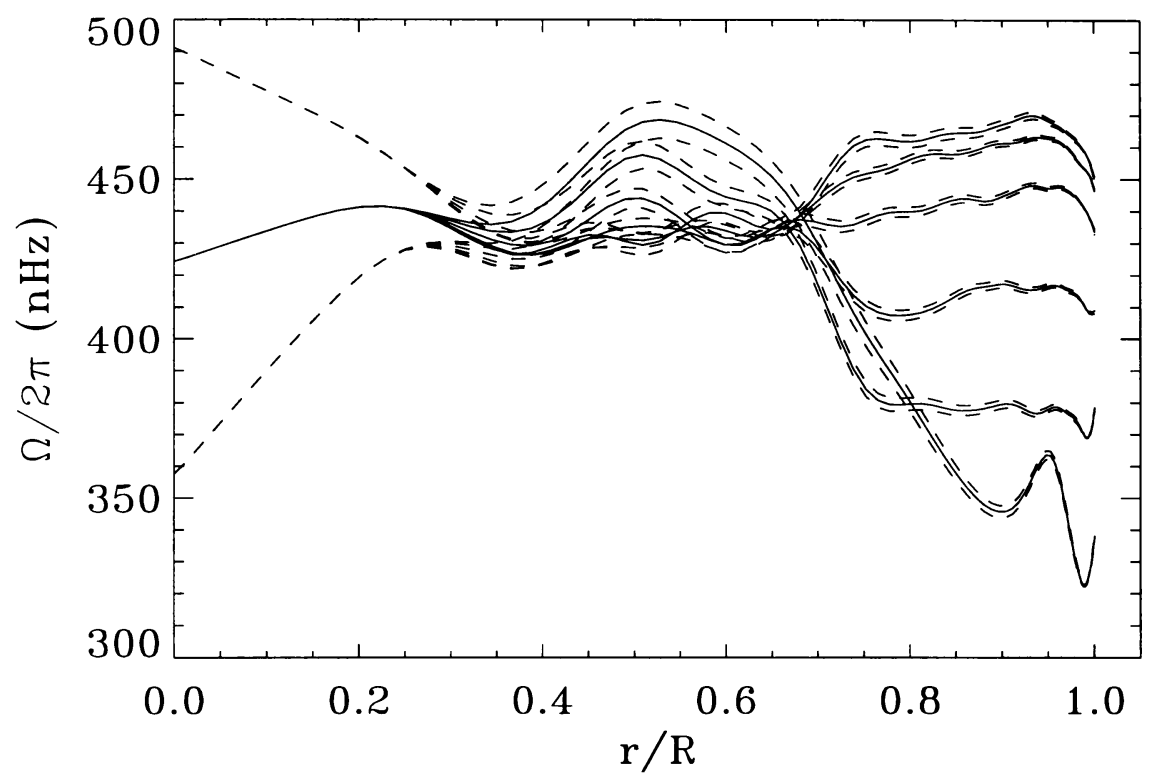

Figure 3. Plot of the rotation rate as a function of radius for a number of latitudes. Latitudes are from top to bottom near the solar surface: $0^{\circ}, 15^{\circ}, 30^{\circ}, 45^{\circ}, 60^{\circ}$ and $75^{\circ}$. One $\sigma$ error bars have been indicated by dashed lines. Results are from the inversion shown in Fig. 1.

Also seen in Fig. 3 is the rapid increase in the rotation rate as one goes inwards close to the equator. Partly because of the many $f$ modes available in the dataset, we have been able to obtain significantly improved results close to the surface. This increase near the surface may explain the difference between the rotation rates observed using surface Doppler shift and by following features such as sunspots and supergranulation. It is interesting to note that the depth dependence changes as latitude varies, and may even become a decrease at the higher latitudes. The cause of this is not currently clear.

\section{Near-Surface Rotation}

The inferred rotation rate close to the solar surface is dominated by the contributions of the $a_{1}, a_{3}$ and $a_{5}$ terms. Higher order terms are also quite significant, as illustrated by the inversion shown in Fig. 4 , where $a_{1}, a_{3}$ and $a_{5}$ were artificially set to zero. There are small scale variations in the rotation rate reminiscent of the so-called torsional oscillation (Howard \& LaBonte, 1980; Hathaway et al., 1996) seen in surface Doppler shift data. These patterns seem to exist to a significant depth (at least $20 \mathrm{Mm}$ ) as inferred from the weak $l$ dependence of the high-order splittings and from detailed studies of the global inversion results. The results shown in Fig. 4 are most sensitive to the rotation rate between about $2 \mathrm{Mm}$ and $9 \mathrm{Mm}$ below the solar surface. Some of these results were discussed in Kosovichev \& Schou (1997). 

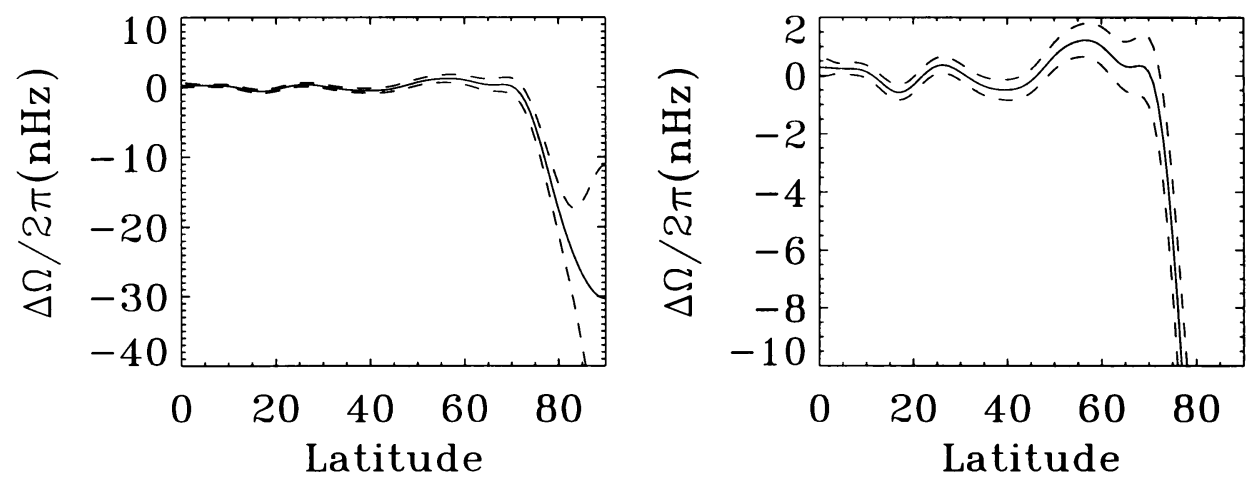

Figure 4. The near-surface rotation rate obtained by setting $a_{1}=a_{3}=a_{5}=0$ and inverting for the latitude dependence (Kosovichev \& Schou, 1997). Only splittings from f-modes with $88 \leq l \leq 250$ were used. $10 \sigma$ error bars are indicated by dashed lines. The results above $75-80^{\circ}$ latitude are unlikely to be reliable.

Another feature in Fig. 4 is the very slow rotation of the polar regions. This can also be seen in the global inversion in Figs. 1 and 3. This "polar vortex" seems to extend to a significant depth. Again this is close to the edge of the reliable region and it is unclear whether it is related to the 'jet'.

Since the surface Doppler shift signal seems to indicate that the zonal flows migrate to lower latitudes during the solar cycle, five 72 day time-series were analyzed individually. Preliminary results of this analysis, shown in Fig. 5, suggest that the patterns drift towards lower latitudes in a manner similar to the drift of the torsional oscillation signal and the drift in the latitude of emergence of active regions during the solar cycle (as seen in the butterfly diagram).

It is important to note that this signal is an average down to a substantial depth, not simply a surface measurement. Earlier suggestions that the observed surface Doppler shift signal could be due to some sort of systematic error caused by the magnetic fields would thus seem to be ruled out.

\section{Conclusion}

Recently available datasets from a variety of instruments have allowed us to probe the solar internal rotation in considerably more detail than previously possible. We have succeeded in constraining the tachocline at the bottom of the solar convection zone; we have been able to measure small scale features in the solar rotation rate near the surface; we have detected variations in the near surface rotation rate and we have seen hints of a jet in the convection zone at high latitude.

It is, however, also clear that we have not fully exploited the new datasets and that further discoveries are likely to be made in the near future. In particular we hope to be able to detect other solar cycle variations in addition to the drift of the small scale zonal flows, obtain better constraints on the core rotation and further constrain the properties of the tachocline. It has been suggested that the position and width 


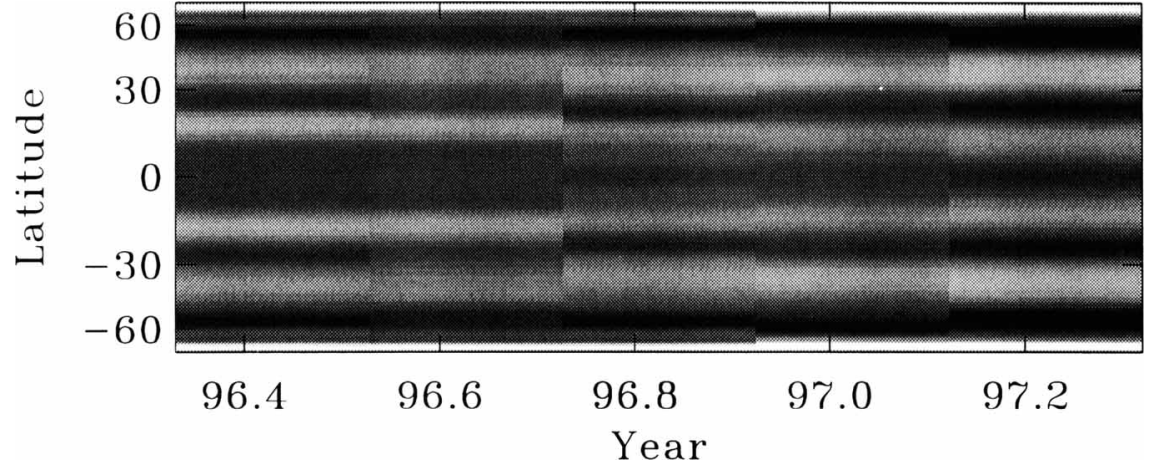

Figure 5. The near surface rotation rate, inferred as in Fig. 4, for five different 72 day time-series. The results for each time period have been shown as a grey scale plot with white being $5 \mathrm{~m} / \mathrm{s}$ slower rotation and black $5 \mathrm{~m} / \mathrm{s}$ faster. Due to the shorter duration of the individual time-series and the desire to use the same set of modes for each period, the set of modes used for this plot is smaller than that used for Fig. 4.

of the tachocline may vary with latitude and that the variation might be detectable by current datasets or those available in the near future. Such a latitude dependence could be detectable both in the rotation rate and in the solar sound speed using the even $a$-coefficients. One might also hope that it will be possible to detect the smallscale features found close to the surface at greater depths and to detect the possible motion of the jet.

To achieve some of these goals it is clear that we will need observations over longer periods of time, preferably at least one solar activity cycle. Fortunately several instruments, including MDI, GONG and LOWL, are likely to continue operations for several years. Each project requires, of course, continued funding and, especially in the case of space based instruments, the absence of fatal technical problems. Due to the often subtle nature of the data analysis and interpretation, it is also highly desirable to check results using different instruments and analysis techniques, when feasible.

\section{Acknowledgements}

The 'SOI internal rotation team' co-authors are the co-authors of Schou et al. (1998), viz, H.M. Antia, S. Basu, R.S. Bogart, R.I. Bush, S.M. Chitre, J. Christensen-Dalsgaard, A. Eff-Darwich, D.O. Gough, J.T. Hoeksema, R. Howe, S.G. Korzennik, A.G. Kosovichev, R.M. Larsen, F.P. Pijpers, P.H. Scherrer, T. Sekii, T.D. Tarbell, A.M. Title, M.J. Thompson and J. Toomre.

The MDI project is supported by NASA contract NAG5-3077 at Stanford University. SOHO is a mission of international cooperation between the ESA and NASA. 


\section{References}

Basu, S. (1997) Monthly Notices Roy. Astron. Soc. 288, 572.

Charbonneau, P. et al. (1997) in J. Provost and J. Schmider (eds.), IAU Symp. 181: Sounding Solar and Stellar Interiors, Kluwer, Dordrecht, Holland, in press.

Duvall, T. L., Jr. et al. (1984) Nature 310, 22.

Christensen-Dalsgaard, J. \& Schou, J. (1988) in E. J. Rolfe (ed.), ESA SP-286: Seismology of the Sun \& Sun-Like Stars, p. 149.

Giles, P. M. \& Duvall, T. L., Jr. (1998) these proceedings, in press.

Giles, P. M., Duvall, T. L. Jr., Scherrer, P. H., and Bogart, R. S. (1997) Nature, in press.

Hathaway, D. H. et al. (1996), Science 272, 1306.

Howard, R. \& LaBonte, B. J. (1980), Astrophys. J. 239, L33.

Kosovichev, A. G. (1996) Astrophys. J. 469 L61.

Kosovichev, A. G. \& Schou, J. (1997) Astrophys. J. 482, L207.

Schou, J., et al. (1998) Astrophys. J. in preparation.

Schou, J., Christensen-Dalsgaard, J. and Thompson, M. J. (1994) Astrophys. J. 433, 389.

Thompson, M. J. (1998) these proceedings, in press.

Thompson, et al. (1996) Science 272, 1300.

Tomczyk, S., Schou, J. and Thompson, M. J. (1995) Astrophys. J. 448, L57. 